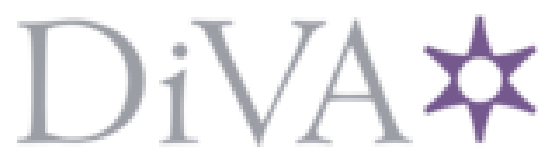

http://www.diva-portal.org

This is the published version of a paper published in Physical Review A. Atomic, Molecular, and Optical Physics.

Citation for the original published paper (version of record):

Linusson, P., Hedin, L., Eland, J H., Squibb, R J., Mucke, M. et al. (2013)

Complete double valence photoionization study of the electron spectra of krypton.

Physical Review A. Atomic, Molecular, and Optical Physics, 88(2): 022510

http://dx.doi.org/10.1103/PhysRevA.88.022510

Access to the published version may require subscription.

N.B. When citing this work, cite the original published paper.

Permanent link to this version:

http://urn.kb.se/resolve?urn=urn:nbn:se:uu:diva-207526 


\title{
Complete double valence photoionization study of the electron spectra of krypton
}

\author{
P. Linusson, ${ }^{1}$ L. Hedin, ${ }^{2}$ J. H. D. Eland, ${ }^{3,2}$ R. J. Squibb,${ }^{2}$ M. Mucke, ${ }^{2}$ S. Zagorodskikh, ${ }^{2}$ L. Karlsson, ${ }^{2}$ and R. Feifel ${ }^{2}$ \\ ${ }^{1}$ Department of Physics, Stockholm University, AlbaNova University Center, SE-106 91 Stockholm, Sweden \\ ${ }^{2}$ Department of Physics and Astronomy, Uppsala University, Box 516, SE-751 20 Uppsala, Sweden \\ ${ }^{3}$ Department of Chemistry, Physical and Theoretical Chemistry Laboratory, Oxford University, South Parks Road, \\ Oxford OX1 3QZ, United Kingdom
}

(Received 3 June 2013; published 21 August 2013)

\begin{abstract}
Double photoionization spectra of $\mathrm{Kr}$ have been recorded using monochromatized synchrotron radiation of $88 \mathrm{eV}$ photon energy and a versatile multielectron coincidence time-of-flight spectroscopy technique. The formation of the $\mathrm{Kr}^{2+}$ states of the lowest-energy configuration $4 s^{2} 4 p^{4}$ is partly direct, producing electron pairs with a continuous distribution, and partly indirect via superexcited singly ionized states. The superexcited $\mathrm{Kr}^{+}$ states show strong and hitherto unexplained selectivity in branching to final $\mathrm{Kr}^{2+}$ states. $\mathrm{Kr}^{2+}$ states based on excited configurations are formed mainly by direct double photoionization.
\end{abstract}

DOI: 10.1103/PhysRevA.88.022510

PACS number(s): 33.70.Ca, 33.80.Eh, 34.50.Gb

\section{INTRODUCTION}

Double ionization of atoms and molecules induced by single-photon absorption has been the subject of intense research for many years. A major focus of interest has been direct double ionization, meaning a simultaneous release of two electrons, in a process requiring strong electron correlation. The indirect double photoionization process, in which an intermediate singly ionized state is first formed (by a one-photon two-electron transition) and then decays by autoionization to a doubly charged ion, has been much less well studied. It is only with the advent of multicoincidence techniques that the second step, autoionization, in indirect double photoionization can be studied directly and the branching of particular monocationic levels to final dicationic states determined. Experimentally, the $n p^{-2}$ double ionization of the noble gases has been explored to a considerable extent (see, e.g., Refs. [1-4], and references therein). Double photoionization (DPI) leading to higher excited states of the dications, e.g., with a vacancy in the outermost $s$ subshell, has received comparatively less attention. So far, neon has been thoroughly explored and the cross section for the formation of states related to the $2 s^{1} 2 p^{5}$ and $2 s^{0} 2 p^{6}$ configurations has been determined by both optical spectroscopy [5] and electron-electron coincidence spectroscopy [6]. Threshold photoelectron coincidence (TPEsCO) [7] studies have been carried out for photon energies covering the entire region below the core levels in neon and argon [8], as well as in xenon [9].

In this work, we present an electron-electron coincidence study of doubly ionized krypton atoms, using the time-offlight magnetic bottle spectrometer technique with full energy multiplexing (Ref. [3], and references therein) at a photon energy of $88 \mathrm{eV}$. This is sufficient for the formation of dicationic states with energies up to and including the $4 s^{0} 4 p^{6}$ ${ }^{1} S$ state, but below the binding energy of the $3 d$ core levels. Assignments of most of the lines, based upon data from high-resolution optical spectroscopy collected in the NIST database [10] and upon Auger electron spectroscopy studies $[11,12]$, provide a much more complete characterization of the process than before. At the chosen photon energy, the $\mathrm{Kr}$ photoelectron spectrum [13] shows a strong $\left({ }^{1} D\right) n d{ }^{2} S$ series of satellites, as well as a complicated satellite spectrum in the binding-energy region above $40 \mathrm{eV}$. Their influence on the formation of the states derived from the $4 p^{4}$ ground-state configuration is investigated in detail and shows remarkable selectivity in the autoionization branching.

\section{EXPERIMENTAL DETAILS}

The experiments were carried out using pulsed monochromatized synchrotron radiation as available at beam line U49/2 PGM-1 of the BESSY-II storage ring in Berlin, Germany. The storage ring was operated in single-bunch mode, providing light pulses at a rate of approximately $1.25 \mathrm{MHz}$. The magnetic bottle Time-Of-Flight (TOF) PhotoElectron-PhotoElectron COincidence (PEPECO) technique was used to measure the energies of all electrons liberated by single photons. Its principle has been reported earlier [4]. For the present studies, a 2.2-m-long magnetic bottle flight tube has been employed to determine the flight time of each of the two electrons ejected upon double ionization and detected in coincidence. Due to the long flight tube, near-zero kinetic-energy electrons have a typical flight time of $\sim 4 \mu \mathrm{s}$, while those electrons with kinetic energies in excess of $\sim 21 \mathrm{eV}$ have TOFs shorter than the inter-light-pulse period ( $~ 800.5 \mathrm{~ns})$. This means that the light pulses provided by the BESSY-II storage ring, even when operated in single bunch mode, do not provide an absolute time reference in particular for low-kinetic-energy electrons. For unambiguous determination of the TOF of the electrons, the light pulse frequency was therefore reduced to $\sim 78 \mathrm{kHz}$ by means of a mechanical chopper [14]. The electron counting rate was held at approximately $3 \mathrm{kHz}$ and the photon energy resolution was below $10 \mathrm{meV}$. The sample gas was obtained commercially with a stated purity of better than $99 \%$.

\section{RESULTS AND DISCUSSION}

Figure 1 shows an electron-electron coincidence map of krypton ionized by $88 \mathrm{eV}$ photons, where the kinetic energy of the first arrival electron (fast electron) is plotted versus the kinetic energy of the second arrival electron (slow electron). The intensity in the map is distributed along the lines of the constant kinetic-energy sum of two electrons, which correspond to states of the dication, where a lower kinetic 


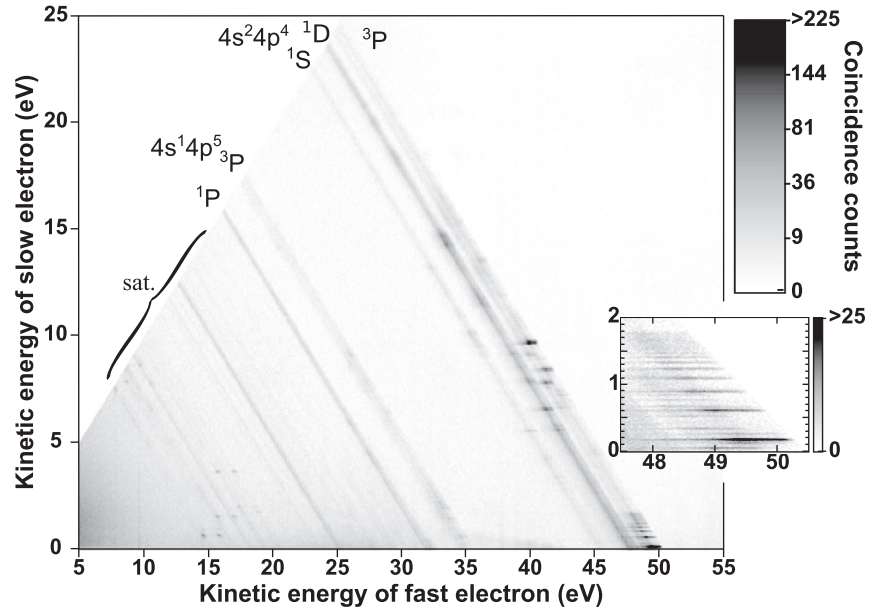

FIG. 1. An electron-electron coincidence map of krypton ionized by $88 \mathrm{eV}$ photons. The grayscale indicates the intensity (coincidence counts $)$ of a channel $(50 \times 50 \mathrm{meV}$ for the full map, $10 \times 10 \mathrm{meV}$ for the inset). To enhance weak features, the intensity in the map is scaled by taking its square root; in addition, an intensity above 225 is drawn as dark black. In the lower left corner of the map, spots of intensity are seen which originate from second- and third-order light, as discussed in the text.

sum corresponds to a higher double-ionization energy. The intensity distribution reveals how the excess energy is shared between the two electrons. A dark spot on the map represents double ionization via an intermediate singly ionized state, as in that case the kinetic energies of both electrons are fixed, within a narrow range if lifetime broadening of the intermediate state is negligible. Diagonal lines in the map indicate substantial direct double ionization, as the excess energy can be shared in a continuous manner. As can be seen in Fig. 1, in general both indirect and direct double-ionization pathways are possible, but indirect ionization tends to contribute less to the intensity at higher ionization energies.

\section{A. The Kr double-ionization spectrum}

Figure 2 shows the complete electron spectrum of doubly ionized $\mathrm{Kr}$ states between 35 and $75 \mathrm{eV}$ ionization energy. An asymmetry in the peaks in Fig. 2 is noted and attributed to a spectrometer effect, most notable for electrons with comparatively high kinetic energies. In view of this, the maxima of the observed peaks in the double-ionization spectrum are given in Table I, along with peak areas and assignments. Because of the difficulties encountered in properly modeling the peak shapes, we expect the extracted areas to be accurate only to within $10 \%$ for the most intense peaks. For the weaker peaks at higher $(>55 \mathrm{eV})$ ionization energy, a nonlinear background prevents an additional complication and hence the reported intensities of these peaks are mostly indicative.

The first group of lines is located in the $35-45 \mathrm{eV}$ region. The lines correspond to the $4 p^{4}\left({ }^{3} P_{2,1,0} ;{ }^{1} D_{2} ;{ }^{1} S_{0}\right)$ finestructure levels of the dication. The first line is comparatively broad and is associated with the ${ }^{3} P_{2,1,0}$ levels, which are partly resolved. The next two lines, which are well separated, correspond to the ${ }^{1} D_{2}$ and ${ }^{1} S_{0}$ states of this configuration.

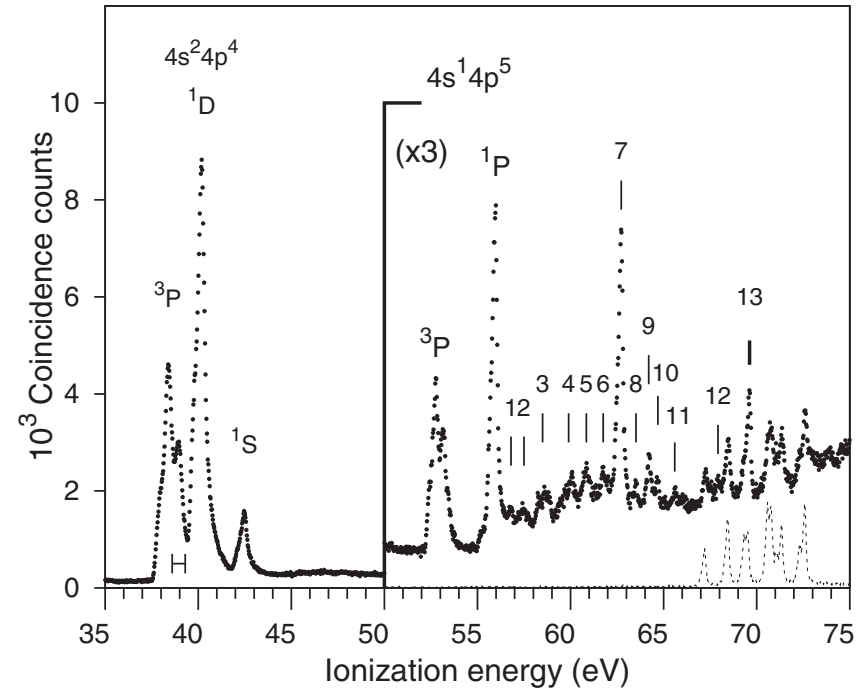

FIG. 2. An overview of the valence double-ionization electron spectrum of Kr. A horizontal line with bars indicates the energy region used for the identification of processes leading to the formation of the $4 p^{4}{ }^{3} P_{1,2}$ final state, as discussed in the text. Contribution to the spectrum from double Auger decay of $3 d$ core hole states ionized by second- and third-order synchrotron light is indicated by the dashed line. The step size is $25 \mathrm{meV}$.

Above $50 \mathrm{eV}$ ionization energy, a large number of features can be observed. The first two strong peaks located at around 53 and $56 \mathrm{eV}$ can be readily assigned to the ${ }^{3} P$ and ${ }^{1} P$ states of the $4 s 4 p^{5}$ configuration. As for the $4 p^{4}{ }^{3} P$ states, the fine

TABLE I. Ionization energies (peak maxima) and intensities of doubly ionized states of $\mathrm{Kr}$ at the photon energy of $88.0 \mathrm{eV}$. Peak numbers refer to Fig. 2. In some cases, several states are energetically possible (cf. Ref. [10]), as indicated by “...". For brevity, the prominent states are listed in those cases.

\begin{tabular}{|c|c|c|c|}
\hline Peak no. & Energy $(\mathrm{eV})$ & Assignment & Rel. Int. \\
\hline & 38.40 & $4 s^{2} 4 p^{4}{ }^{3} P_{2}$ & 67 \\
\hline & 39.93 & $4 s^{2} 4 p^{4}{ }^{3} P_{1,0}$ & \\
\hline & 40.18 & $4 s^{2} 4 p^{4}{ }^{1} D$ & 100 \\
\hline & 42.46 & $4 s^{2} 4 p^{4}{ }^{1} S$ & 9.5 \\
\hline & 52.76 & $4 s^{1} 4 p^{5}{ }^{3} P_{2}$ & 13 \\
\hline & 53.14 & $4 s^{1} 4 p^{5}{ }^{3} P_{1,0}$ & \\
\hline & 55.96 & $4 s^{1} 4 p^{5}{ }^{1} P$ & 14 \\
\hline 1 & 56.8 & $4 p^{3}\left({ }^{4} S\right) 4 d^{3} D$ & $<0.5$ \\
\hline 2 & 57.5 & $4 p^{3}\left({ }^{2} D\right) 4 d{ }^{3} F ;{ }^{1} S$ & 1 \\
\hline 3 & 58,59 & $\begin{array}{c}4 p^{3}\left({ }^{2} D\right) 4 d^{3} G ;{ }^{1} G \\
5 s^{3} D ; 4 p^{3}\left({ }^{2} P\right) 4 d^{1} D\end{array}$ & 3 \\
\hline 4 & 60 & $4 p^{3}\left({ }^{2} P\right) 4 d^{3} P ;{ }^{3} F ; \ldots$ & 3 \\
\hline 5 & 60.9 & $4 p^{3}\left({ }^{2} P\right) 5 s^{1} P ; \ldots$ & 2 \\
\hline 6 & 61.7 & $4 p^{3}\left({ }^{2} D\right) 4 d^{3} P ; \ldots$ & 1 \\
\hline 7 & 62.72 & $4 p^{3}\left({ }^{2} D\right) 4 d{ }^{1} P_{1} ; \ldots$ & 10 \\
\hline 8 & 63.5 & $4 p^{3}\left({ }^{2} D\right) 5 p{ }^{1} D ; \ldots$ & $<0.5$ \\
\hline 9 & 64.2 & $\begin{array}{c}4 p^{3}\left({ }^{2} P\right) 5 p^{3} D ;{ }^{3} P \\
4 p^{3}\left({ }^{2} D\right) 5 p{ }^{3} S ; \ldots\end{array}$ & 2 \\
\hline 10 & 64.7 & $4 p^{3}\left({ }^{2} P\right) 4 d{ }^{1} P ;{ }^{1} D ; \ldots$ & 1 \\
\hline 11 & 65.6 & & 1 \\
\hline 12 & 67.9 & & $<0.5$ \\
\hline 13 & 69.6 & $4 s^{0} 4 p^{6}{ }^{1} S$ & 2 \\
\hline
\end{tabular}


structure of $4 s 4 p^{5}{ }^{3} P$ is only partially resolved. At higher ionization energies, the spectrum becomes highly complex, as excited configurations of the type $4 p^{3} n l$ give rise to many possible states. At around $62.7 \mathrm{eV}$ ionization energy, an intense peak is observed. From studies of the $M-N N$ Auger decay [15], it is known that strong configuration interaction (CI) between $4 p^{3} 4 d$ and $4 s 4 p^{5}$ causes large redistribution of the intensity for the $J=1$ levels, and therefore we attribute line 7 to be a $4 p^{3} 4 d^{1} P_{1}$ satellite. Numerous other, less intense features are also observed in the energy region 52-65 eV. As individual peaks are not resolved, it is difficult to determine if there is a systematic behavior of the intensities. It appears (e.g., structure 3$)$ that states with high $J$ values $(J>2)$, whose intensity cannot be explained by CI with the $4 s 4 p^{5}$ configuration, also acquire non-negligible intensities.

At ionization energies above $65 \mathrm{eV}$, five moderately strong peak structures are observed. Most of the intensity likely appears because of spectral artifacts originating from the double Auger (DA) decay of the $3 d$ core holes $[16,17]$ ionized by second- and third-order synchrotron light. While a triple ionization event in reality, the DA electrons appear in the two-electron coincidence data when the photoelectron is not detected. Their contribution to the intensity of the doubleionization spectrum can be estimated from triple electron coincidences, when the $3 d$ photoelectron is detected, and is indicated in Fig. 2. However, part of feature 13 comes from double ionization and can be assigned to the $4 s^{0} 4 p^{6}{ }^{1} S$ state [10]. We note that this assignment disagrees with Ref. [15], but is supported by a recent calculation by Pernpointer et al. [18].

\section{B. Double-ionization processes}

The relative areas of the three outermost line structures reflect the probabilities of creating the ${ }^{3} P,{ }^{1} D$, and ${ }^{1} S$ states. The observed intensity ratios of 7.1:10.6:1, as obtained from the peak areas, are different compared to previous experimental observations using ionizing radiation from He II [4]. Part of the explanation is contributions to the intensity from indirect double ionization via intermediate singly ionized states. Obviously, some of them were not energetically accessible in the work of Ref. [4] and, in addition, those that were appear to be of less importance at the present photon energy.

In Fig. 3(a), the low-kinetic-energy part of the electronenergy distribution associated with the formation of the $4 p^{4}$ ${ }^{3} P_{2,1,0}$ states is shown. Line positions and relative intensities of the features observed are given in Table II. The observed peaks arise because of autoionization to the ${ }^{3} P$ states; for comparison with photoelectron spectroscopy (PES) studies, one of the two energy scales represents the electron kinetic energy plus the formation energy for the ${ }^{3} P_{2}$ ground state of the dication. Here and subsequently, we assume an energy of $38.3594 \mathrm{eV}$ for the ${ }^{3} P_{2}$ state according to the NIST database [10]. At the present photon energy of $88 \mathrm{eV}$, the most intense satellite features in the $\mathrm{Kr}$ photoelectron spectrum in the binding energy region $38-40 \mathrm{eV}$ are attributed to the $\left({ }^{1} D\right) n d{ }^{2} S$ states $[13,19,20]$. In the autoionization spectrum of Fig. 3(a), this is manifested by a series of strong peaks $(3,7,11,13,15-18)$ corresponding to $\left({ }^{1} D\right) n d{ }^{2} S \rightarrow{ }^{3} P_{2}$ transitions. A copy of this series (peaks $1,5,6,8-10)$ can also be discerned for $n=8-13$, which differs for each $n$ in energy to the original very nearly by the ${ }^{3} P_{2,1}$ level
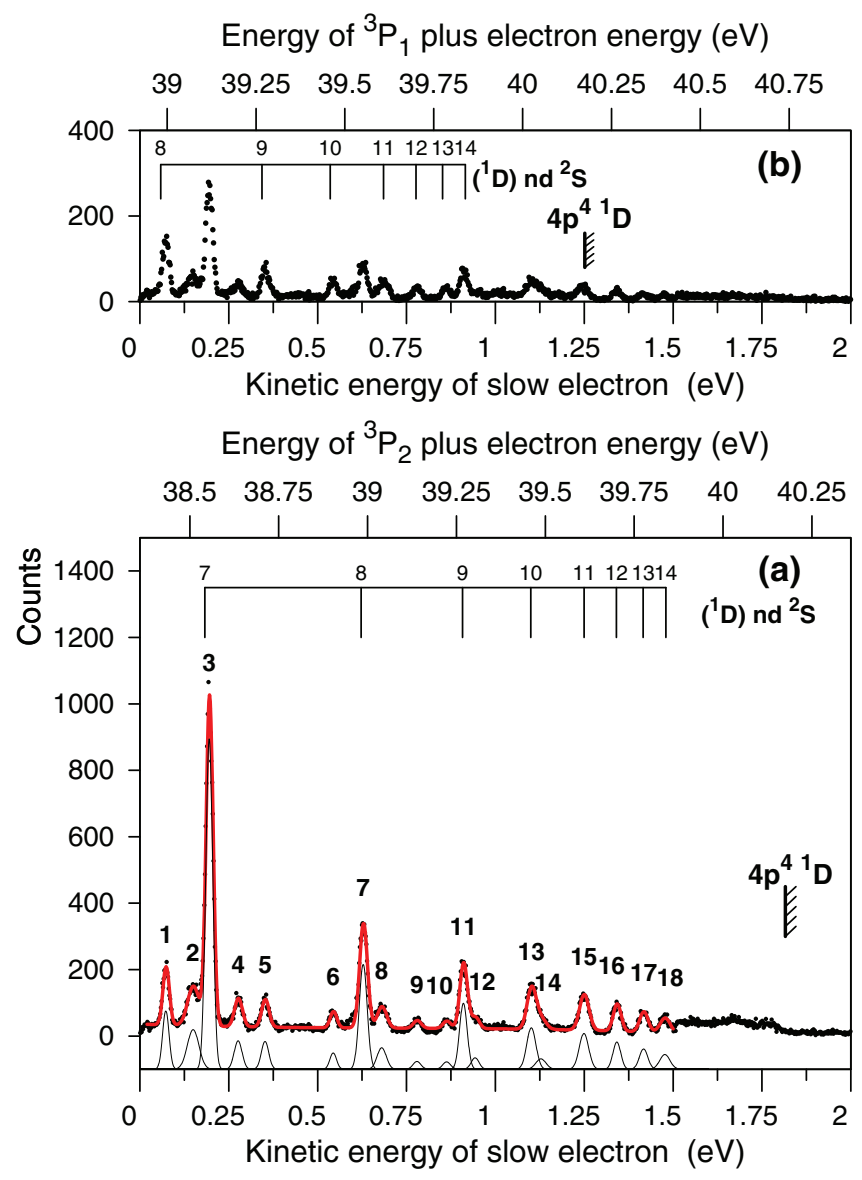

FIG. 3. (Color online) Spectra of electrons released in the formation of the $4 p^{4}{ }^{3} P$ states obtained from different selections on the kinetic-energy sum of the two electrons. (a) The entire peak (cf. Fig. 2) corresponding to the ${ }^{3} P$ states has been selected. Dots show experimental results and solid lines represent a fit to Gaussian peak shapes. The top scale is the electron kinetic energy plus the energy of the ${ }^{3} P_{2}$ ground state of the dication relative to neutral $\mathrm{Kr}$. (b) A subset of the data in an energy region (indicated in Fig. 2) corresponding to the ${ }^{3} P_{1,0}$ final states has been selected. The step size is $2 \mathrm{meV}$ in both (a) and (b).

splitting in the dication. The relative intensity of the repeated series is enhanced in Fig. 3(b), where we have selected a subset of the coincidence data according to an energy which corresponds to the formation of the ${ }^{3} P_{1,0}$ final states. We note that overlap with the decay to the ${ }^{3} P_{2}$ state occurs, in particular, for $n=11,14$. In addition, a weak feature at a binding energy approximately corresponding to peak 1 in Fig. 3(a) was observed in a photoelectron spectrum recorded at $68.5 \mathrm{eV}$ by Caló et al. [20]. However, it was not reported in the $125 \mathrm{eV}$ study by Alitalo et al. [19], and we note that peak 5 in Fig. 3(a) has no correspondence in the photoelectron spectra of either study. In view of these observations, we assign the repeated series as dominated by $\left({ }^{1} D\right) n d{ }^{2} S \rightarrow{ }^{3} P_{1}$ autoionization and estimate a branching ratio of the decay of the $\left({ }^{1} D\right) n d{ }^{2} S(n=8-10)$ singly ionized states to the ${ }^{3} P$ $J=2 ; 1 ; 0$ levels of the dication to be approximately $2.2 ; 1 ; \sim 0$. Further experimental effort is required to deduce more accurate 
branching ratios, as it is not clear from the present results whether this ratio changes for higher $n$.

It is interesting to compare the present results with the $n p^{4}\left({ }^{1} D\right) n d{ }^{2} S$ autoionizing resonances in the halogens, particularly $\mathrm{Br}$ which is isoelectronic to $\mathrm{Kr}^{+}$. In strict LS coupling, the autoionization of the $\left({ }^{1} D\right) n d{ }^{2} S$ Rydberg series in the halogens to the ${ }^{3} P$ states is "forbidden," but occurs as a consequence of spin-orbit interaction [21] as the $\left({ }^{1} D\right) n d{ }^{2} S$ resonance states can contain small admixtures of $\left({ }^{1} D\right) n d{ }^{2} P_{1 / 2}$ [22] and the "target" ${ }^{3} P_{2}$ and ${ }^{1} D_{2}$ cores are coupled [23]. Autoionization to the ${ }^{3} P_{2}$ final state was found experimentally to be dominant for the $\left({ }^{1} D\right)$ nd ${ }^{2} S$ resonances in $\mathrm{Cl}$ and $\mathrm{Br}$ [24,25], and only weak transitions to the $J=1,0$ levels were observed. The apparently much smaller ${ }^{3} P_{2} /{ }^{3} P_{1}$ branching ratio observed here for the decay of the $\mathrm{Kr}^{+}$states compared to $\mathrm{Br}$ may, to some extent, be explained by the increased effective charge of the nucleus, increasing spin-orbit effects. Another obvious difference is the presence of an escaping photoelectron in the present case so that coupling between the continuum channels should be considered. However, estimating the importance of such couplings will require theoretical investigations which

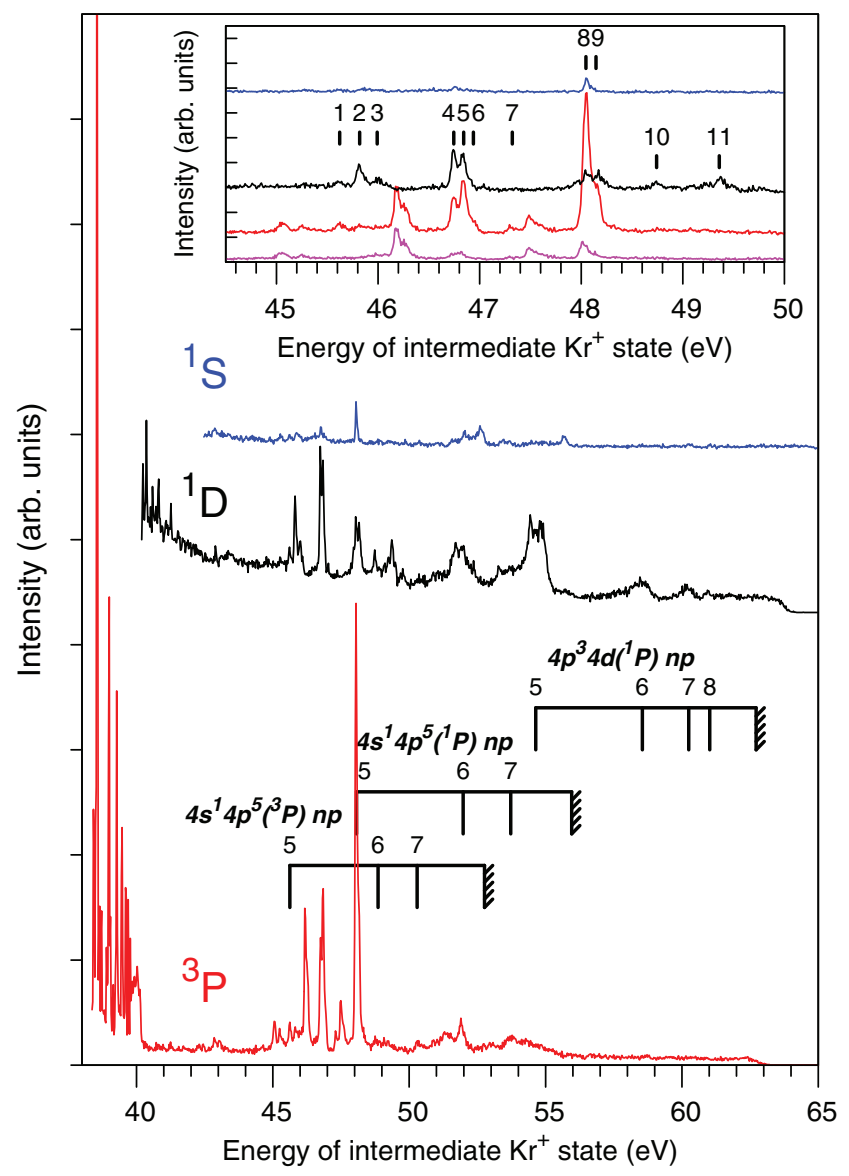

FIG. 4. (Color online) Spectrum of electrons released in the formation of the $4 p^{4}{ }^{3} P,{ }^{1} D$, and ${ }^{1} S$ states of the $\mathrm{Kr}$ dication. For the lowest trace in the inset, which is essentially a zoomed-in version of the main figure, a final-state energy region corresponding to the formation of the ${ }^{3} P_{1,0}$ states (cf. Fig. 3) has been selected. The energy scale is the ionization energy of the indicated state plus the electron kinetic energy. The intensity scale is common, but traces have been offset vertically.
TABLE II. Line positions and intensities observed in the low kinetic energy $(<2 \mathrm{eV})$ formation spectrum of the $4 p^{4}{ }^{3} P$ states. The last column denotes binding energies (BE). The error in relative energies is estimated to be within $10 \mathrm{meV}$. The relative intensities (Rel. Int.) are expected to be accurate within $10 \%$ unless otherwise stated (in form of giving the actual deviations as numbers in parentheses).

\begin{tabular}{lcccc}
\hline \hline Line & $\mathrm{E}_{\text {kin }}(\mathrm{eV})$ & Rel. Int. & Decay & $\mathrm{BE}(\mathrm{eV})$ \\
\hline 1 & 0.073 & 16.3 & ${ }^{1} D 8 d{ }^{2} S \rightarrow{ }^{3} P_{1}$ & 38.996 \\
2 & 0.150 & 20.0 & ${ }^{1} S 6 p \rightarrow{ }^{3} P_{2}$ & 38.509 \\
3 & 0.195 & 100 & ${ }^{1} D 7 d{ }^{2} S \rightarrow{ }^{3} P_{2}$ & 38.555 \\
4 & 0.276 & 9.7 & ${ }^{1} D 8 d{ }^{2} D_{3 / 2} \rightarrow{ }^{3} P_{2}$ & 38.636 \\
5 & 0.352 & 8.9 & ${ }^{1} D 9 d{ }^{2} S \rightarrow{ }^{3} P_{1}$ & 39.275 \\
6 & 0.544 & 4.7 & ${ }^{1} D 10 d{ }^{2} S \rightarrow{ }^{3} P_{1}$ & 39.468 \\
7 & 0.628 & 35.9 & ${ }^{1} D 8 d{ }^{2} S \rightarrow{ }^{3} P_{2}$ & 38.988 \\
8 & 0.680 & 8.6 & ${ }^{1} D 11 d^{2} S \rightarrow{ }^{3} P_{1}$ & 39.604 \\
9 & 0.780 & $2.4(5)$ & ${ }^{1} D 12 d^{2} S \rightarrow{ }^{3} P_{1}$ & 39.703 \\
10 & 0.863 & $2.3(5)$ & ${ }^{1} D 13 d{ }^{2} S \rightarrow{ }^{3} P_{1}$ & 39.787 \\
11 & 0.910 & 21.8 & ${ }^{1} D 9 d^{2} S \rightarrow{ }^{3} P_{2}$ & 39.269 \\
12 & 0.943 & 3.9 & ${ }^{1} D 8 d{ }^{2} D_{3 / 2} \rightarrow{ }^{3} P_{2}$ & 39.291 \\
13 & 1.101 & 17 & ${ }^{1} D 10 d{ }^{2} S \rightarrow{ }^{3} P_{2}$ & 39.460 \\
14 & 1.13 & $5(1)$ & ${ }^{1} D 10 d{ }^{2} D_{3 / 2} \rightarrow{ }^{3} P_{2,1}$ & 39.49 \\
15 & 1.249 & 15 & ${ }^{1} D 11 d^{2} S \rightarrow{ }^{3} P_{2}$ & 39.609 \\
16 & 1.342 & 9 & ${ }^{1} D 12 d{ }^{2} S \rightarrow{ }^{3} P_{2}$ & 39.701 \\
17 & 1.417 & 8 & ${ }^{1} D 13 d{ }^{2} S \rightarrow{ }^{3} P_{2}$ & 39.776 \\
18 & 1.477 & 7 & ${ }^{1} D 14 d^{2} S \rightarrow{ }^{3} P_{2}$ & 39.836 \\
\hline \hline
\end{tabular}

are beyond the scope of the present work. We note, as already pointed out in Ref. [4], that a weak decay to the ${ }^{3} P_{0}$ final state can be more readily explained, as the ${ }^{3} P_{0} \in d$ continua are not allowed for a $J=1 / 2$ state and hence a change of angular momentum for the Rydberg electron would be required.

A number of weaker features, peaks 2 and 4 , and the partly resolved peaks 12 and 14 are also seen in Fig. 3(a). Assignments of them are not clear cut, as many states may contribute [19,26], but in the study of Caló et al. [20] the previous assignment [19] of a ${ }^{1} S 6 p$ shakeup state at a binding energy close to that of peak 2 was confirmed, and new assignments of $\left({ }^{1} D\right) n d{ }^{2} D_{3 / 2}$ states close to that of peaks 12 and 14 were made. More information on states near the apparent binding energy of peak 4 was, unfortunately, not reported in the work of Caló et al. [20], but we note that it has an apparent binding energy close to the $\left({ }^{1} D\right) 8 d^{2} D_{3 / 2}$ state, which appears strongly in threshold photoelectron spectra $[27,28]$ and (probably) at the He II photon energies [4]. Hence, we tentatively assign it to that state.

In Fig. 4, the distributions for the formation of the states related to the $4 p^{4}$ configuration are shown on a singleionization energy scale. The photoelectron spectrum of $\mathrm{Kr}$ in the ionization energy region $42-65 \mathrm{eV}$ was included already in the study by Kikas et al. [13] and is very complex. The many overlapping spectral features make assignments difficult. However, below $50 \mathrm{eV}$ ionization energy, some clear lines can be distinguished, which are summarized in Table III along with estimates of the branching ratios of the decay. In the distribution for the ${ }^{1} D$ state, some sharp peaks are also observed for the 40-42 eV binding-energy range, but detailed 
TABLE III. Line positions, relative intensities, and autoionization branching ratios in the binding-energy region $44-50 \mathrm{eV}$. Line numbers refer to Fig. 4.

\begin{tabular}{|c|c|c|c|c|c|c|c|}
\hline \multirow[b]{2}{*}{ Line } & \multirow[b]{2}{*}{$\mathrm{BE}(\mathrm{eV})$} & \multirow[b]{2}{*}{ Assignment (Ref. [13]) } & \multirow[b]{2}{*}{ Rel. Int. (arb. units) } & \multicolumn{4}{|c|}{ Branching $(\%)$} \\
\hline & & & & ${ }^{3} P_{2}$ & ${ }^{3} P_{1,0}$ & ${ }^{1} D_{2}$ & ${ }^{1} S_{0}$ \\
\hline 1 & 45.6 & $4 s 4 p^{5}\left({ }^{3} P\right) 5 p$ & 7 & 44 & 56 & & \\
\hline 2 & 45.8 & & 18 & & 28 & 72 & \\
\hline 3 & 46.0 & & 12 & & 18 & 82 & \\
\hline 4 & 46.7 & $4 s 4 p^{5}\left({ }^{1} P\right) 5 s$ & 64 & 29 & 37 & 31 & 4 \\
\hline 5 & 46.8 & $4 s 4 p^{5}\left({ }^{3} P\right) 4 d$ & 61 & 48 & 22 & 30 & \\
\hline 6 & 46.9 & & 10 & 49 & 51 & & \\
\hline 7 & 47.3 & & 2 & 100 & & & \\
\hline 8 & 48.0 & $4 s 4 p^{5}\left({ }^{1} P\right) 5 p$ & 100 & 76 & 10 & 8 & 6 \\
\hline 9 & 48.1 & & 50 & 76 & 7 & 17 & \\
\hline 10 & 48.7 & & 3 & & & 100 & \\
\hline 11 & 49.4 & & 12 & & & 100 & \\
\hline
\end{tabular}

analysis suggests that they should be attributed rather to the ${ }^{3} P$ state, and appear here due to the imperfect final-state selection.

The $4 s 4 p^{5} n p$ states have been studied extensively in the spectator Auger decay of the $3 d^{-1} n p$ optical resonances [15,29-31], and approximate positions are indicated in Fig. 4. Such states can arise due to monopole shakeup accompanying the $4 s$ ionization, which is expected to dominate at high energies. However, in analyzing the corresponding satellite spectrum of argon at $100 \mathrm{eV}$ photon energy, Combet-Farnoux et al. [32] found that electron correlations, in particular in the ground state, were more important in explaining the intensity of the satellites. A similar case can be expected for the present system and a detailed understanding of the distributions shown in Fig. 4 requires theoretical input. The single-configuration notation used here should be regarded as convenient labels; few of the states are expected to be dominated by single-configuration weights [31].

Regardless of the formation process, the selectivity in autoionization of the satellites to the states of the $4 p^{4}$ configuration is striking. The $4 s 4 p^{5} 4 d, 4 s 4 p^{5} 5 s$, and, in particular, $4 s 4 p^{5} 5 p$ satellites all favor autoionization to the $4 p^{4}{ }^{3} P_{2,1}$ states. We note that this differs from the Ar case, where autoionization to the ${ }^{1} D$ final state dominates for the most intense satellites [32]. At higher ionization energies, most of the intensity is observed as autoionization to the ${ }^{1} D$ state. The most prominent example is the series of broad and asymmetric peaks leading up to the double-ionization limit of the intense $4 p^{3} 4 d{ }^{1} P$ satellite (cf. Fig. 2), which is clearly observed in the $4 p^{4}{ }^{1} D$ distribution but is very weak in the ${ }^{3} P$ and ${ }^{1} S$ distributions. Peaks at similar energies do, however, also appear in the electron distributions related to the $4 s 4 p^{5}$ final states (not shown).

The intensity of the direct DPI channel can be estimated from the data by counting the continuum parts of the energy distributions, as has been done in previous works [4,6]. We follow the work of Ref. [6] and estimate the continuum parts by fitting a hyperbolic function to a number of points in the distributions. In the present case, this approach appears reasonable for the $4 p^{4}{ }^{3} \mathrm{P}$ and ${ }^{1} S$ states, as regions comparatively free of resonance structure are apparent at several points in this energy range, but considerable difficulties are met for the ${ }^{1} D$ state, as essentially the whole available energy range shows some involvement of indirect channels and interference can be present. Keeping the limitations of the approach in mind (see Ref. [6]), we estimate the continuum parts to account for $40 \%, 50 \%$, and $70 \%$ of the intensity of the $4 p^{4}{ }^{3} P,{ }^{1} \mathrm{D}$, and ${ }^{1} S$ states, respectively. In a similar way, the continuum parts of the distributions related to the $4 s 4 p^{5}{ }^{3} P$ and ${ }^{1} P$ states account for approximately $60 \%$ and $80 \%$, respectively, of the intensity. The distributions of higher-lying dicationic states appear essentially free of peak structures.

\section{CONCLUSIONS}

The complete double valence photoionization spectrum of krypton has been investigated using the magnetic bottle TOF-PEPECO method. In the formation of the ${ }^{3} P,{ }^{1} D$, and ${ }^{1} S$ states of the $4 p^{4}$ configuration, indirect double ionization via intermediate singly ionized states account for at least half of the total intensity. The autoionization processes show strong selectivity, which cannot be explained on the basis of known propensity rules and thus presents a challenge to existing theory.

\section{ACKNOWLEDGMENTS}

This work has been financially supported by the Swedish Research Council (VR), the Göran Gustafsson Foundation (UU/KTH), the Knut and Alice Wallenberg Foundation, and the Carl Tryggers Foundation, Sweden. We would like to warmly acknowledge the support by the staff and colleagues at BESSY-II, Berlin. This work was also supported by the European Community - Research Infrastructure Action under the FP6 "Structuring the European Research Area" Programme (through the Integrated Infrastructure Initiative "Integrating Activity on Synchrotron and Free Electron Laser Science" - Contract No. R II 3-CT-2004-506008). 
[1] P. Bolognesi, G. C. King, and L. Avaldi, Rad. Phys. Chem. 70, 207 (2004).

[2] G. C. King and L. Avaldi, J. Phys. B: At. Mol. Opt. Phys. 33, R215 (2000).

[3] J. H. D. Eland, Adv. Chem. Phys. 141, 103 (2009).

[4] J. H. D. Eland, O. Vieuxmaire, T. Kinugawa, P. Lablanquie, R. I. Hall, and F. Penent, Phys. Rev. Lett. 90, 053003 (2003).

[5] K. H. Schartner, G. Mentzel, B. Magel, B. Mobus, A. Ehresmann, F. Vollweiler, and H. Schmoranzer, J. Phys. B: At. Mol. Opt. Phys. 26, L445 (1993).

[6] T. Kaneyasu, Y. Hikosaka, E. Shigemasa, F. Penent, P. Lablanquie, T. Aoto, and K. Ito, Phys. Rev. A 76, 012717 (2007).

[7] R. I. Hall, A. McConkey, K. Ellis, G. Dawber, L. Avaldi, M. A. MacDonald, and G. C. King, Meas. Sci. Technol. 3, 316 (1992).

[8] L. Avaldi, G. Dawber, N. Gulley, H. Rojas, G. C. King, R. Hall, M. Stuhec, and M. Zitnik, J. Phys. B: At. Mol. Opt. Phys. 30, 5197 (1997).

[9] P. Bolognesi, S. J. Cavanagh, L. Avaldi, R. Camilloni, M. Zitnik, M. Stuhec, and G. C. King, J. Phys. B 33, 4723 (2000).

[10] Y. Ralchenko, A. Kramida, J. Reader, and NIST ASD Team (2010), NIST Atomic Spectra Database (version 4.0.1), http://physics.nist.gov/asd (National Institute of Standards and Technology, Gaithersburg, MD, 2013).

[11] H. Pulkkinen, S. Aksela, O.-P. Sairanen, A. Hiltunen, and H. Aksela, J. Phys. B: At. Mol. Opt. Phys. 29, 3033 (1996).

[12] H. Aksela, S. Aksela, and H. Pulkkinen, Phys. Rev. A 30, 2456 (1984).

[13] A. Kikas, S. Osborne, A. Ausmees, S. Svensson, O.-P. Sairanen, and S. Aksela, J. Elec. Spec. Rel. Phen. 77, 241 (1996).

[14] S. Plogmaker, P. Linusson, J. H. D. Eland, N. Baker, E. M. J. Johansson, H. Rensmo, R. Feifel, and H. Siegbahn, Rev. Sci. Instrum. 83, 013115 (2012).

[15] H. Aksela, S. Aksela, H. Pulkkinen, G. M. Bancroft, and K. H. Tan, Phys. Rev. A 33, 3876 (1986).
[16] E. Andersson, S. Fritzsche, P. Linusson, L. Hedin, J. H. D. Eland, J.-E. Rubensson, L. Karlsson, and R. Feifel, Phys. Rev. A 82, 043418 (2010).

[17] J. Palaudoux, P. Lablanquie, L. Andric, K. Ito, E. Shigemasa, J.H.D. Eland, V. Jonauskas, S. Kucas, R. Karazija, and F. Penent, Phys. Rev. A 82, 043419 (2010).

[18] M. Pernpointner, J. P. Zobel, and N. V. Kryzhevoi, Phys. Rev. A 85, 012505 (2012).

[19] S. Alitalo, A. Kivimäki, T. Matila, K. Vaarala, H. Aksela, and S. Aksela, J. Elec. Spec. Rel. Phen. 114-116, 141 (2001).

[20] A. Caló, S. Atanassova, R. Sankari, A. Kivimäki, H. Aksela, and S. Aksela, J. Phys. B: At. Mol. Opt. Phys. 39, 4169 (2006).

[21] J. Berkowitz, in Advances in Chemical Physics, edited by I. Prigogine and Stuart A. Rice (Wiley, New York, 2007), Vol. 72, pp. 1-36.

[22] J. E. Hansen, R. D. Cowan, S. L. Carter, and H. P. Kelly, Phys. Rev. A 30, 1540 (1984).

[23] F. Robicheaux and C. H. Greene, Phys. Rev. A 46, 3821 (1992).

[24] S. Benzaid, A. Menzel, J. Jiménez-Mier, S. J. Schaphorst, M. O. Krause, and C. D. Caldwell, Phys. Rev. A 54, R2537 (1996).

[25] S. Benzaid, M. O. Krause, A. Menzel, and C. D. Caldwell, Phys. Rev. A 57, 4420 (1998).

[26] J. Jauhiainen, H. Aksela, O.-P. Sairanen, E. Nõmmiste, and S. Aksela, J. Phys. B: At. Mol. Opt. Phys. 29, 3385 (1996).

[27] M. Hochlaf, H. Kjeldsen, F. Penent, R. I. Hall, P. Lablanquie, M. Lavollé, and J. H. D. Eland, Can. J. Phys. 74, 856 (1996).

[28] H. Yoshii, T. Aoto, Y. Morioka, and T. Hayaishi, J. Phys. B: At. Mol. Opt. Phys. 40, 2765 (2007)

[29] H. Aksela, S. Aksela, A. Mäntykenttä, J. Tulkki, E. Shigemasa, A. Yagishita, and Y. Furusawa, Phys. Scr. T41, 113 (1992).

[30] H. Aksela, J. Jauhiainen, E. Kukk, E. Nõmmiste, S. Aksela, and J. Tulkki, Phys. Rev. A 53, 290 (1996).

[31] J. Mursu, J. Jauhiainen, H. Aksela, and S. Aksela, J. Phys. B: At. Mol. Opt. Phys. 31, 1973 (1998).

[32] F. Combet-Farnoux, P. Lablanquie, J. Mazeau, and A. Huetz, J. Phys. B: At. Mol. Opt. Phys. 33, 1597 (2000). 